\title{
NÚCLEO INTEGRADO DE PROJETOS DE ENGENHARIA (NIPE): INOVAÇÃO QUE ASSOCIA ENSINO, PESQUISA E EXTENSÃO NO IFPA CAMPUS TUCURUÍ
}

\author{
DOI: 10.37702/2175-957X.COBENGE.2021.3364
}

FELIPE RICARDO FARIAS DE SOUSA - felipericardo.engcivil@gmail.com Instituto Federal de Educação do Pará IFPA rua bragança 44 68455-705 - tucurui - PA

Midson César Feitosa Cardoso - midson.cardoso@ifpa.edu.br Instituto Federal de Educação do Pará IFPA

Rua E 102

68458-370 - Tucuruí - PA

Térlys de Araújo Silva - terlys.silva@ifpa.edu.br Instituto Federal de Educação do Pará IFPA

Rua Japurá 28

68455-755 - Tucuruí - PA

HELINEUDES PAIVA DA SILVA - paivatuc@hotmail.com INSTITUTO FEDERAL DO PARÁ

Rua Trrombetas 27

68455-747 - TUCURUI - PA

Resumo: O Núcleo Integrado de Projetos e Engenharia (NIPE) foi idealizado como escritório de projetos, buscando unificar ensino, pesquisa e extensão, cujo funcionamento ocorre em uma carreta acessível, equipada com tecnologia e softwares de amplo uso comercial para produção de projetos de engenharia. Projeto de extensão proposto por alguns professores do Instituto Federal do Pará, Campus Tucuruí, oportuniza estágios para alunos dos cursos técnicos e superiores da própria instituição, e estudantes de engenharia da Universidade Federal do Pará que, a partir dessas tecnologias, elaboram projetos orientados e coordenados por professores engenheiros e pelo engenheiro efetivo do Campus. O presente artigo pretende relatar os objetivos e projetos desenvolvidos no NIPE, bem como demonstrar a importância e eficácia do uso desta metodologia ativa na 
aprendizagem dos alunos sobre engenharia e educação tecnológica, além de agregar e gerar conhecimentos e benefícios em prol da comunidade acadêmica e comunidade em geral. A aplicação da presente metodologia ativa supervisionada pela equipe docente e técnica, observou visível aumento no engajamento dos estudantes e proporcionou enorme melhoria nos resultados práticos de aprendizagem e habilidades transversais, como gestão de tempo, comunicação interpessoal e autonomia nas decisões. Um dos principais pontos positivos observados foi à motivação dos estudantes, no momento em que eles foram capazes de associar os projetos desenvolvidos no escritório com as situações profissionais, conseguindo relacionar teoria \& prática ao contexto real. A interação de equipe possibilitou a conclusão de projetos de engenharia interessantes que serão efetivamente construídos no campus e na própria cidade de Tucuruí, podendo-se afirmar que o NIPE alcançou seus objetivos como meio de fomento em ensino, pesquisa e extensão e geração de estágios e benefícios coletivos.

Palavras-chave: Metodologia Ativa. Ensino por Experimentação. Tecnologia. Escritório. Projetos de Engenharia 


\section{NÚCLEO INTEGRADO DE PROJETOS DE ENGENHARIA (NIPE): INOVAÇÃO QUE ASSOCIA ENSINO, PESQUISA E EXTENSÃO NO IFPA CAMPUS TUCURUÍ}

\section{INTRODUÇÃO}

A expansão do Instituto Federal do Pará (IFPA) no interior do Estado, especificamente no Campus Tucuruí, sudeste do Pará, exige para a manutenção da excelência, constantes avanços metodológicos, associados ao ensino, pesquisa e extensão, na busca por melhores resultados acadêmicos e benefícios para a comunidade. Conforme afirma CUNHA (2018) é necessária uma abordagem metodológica considerando-se os problemas encontrados no mercado de trabalho atual, que exige novas competências além do conhecimento específico, tais como: capacidade de colaboração, conhecimento interdisciplinar, habilidade para inovação, capacidade de trabalho em grupo, inteligência emocional, liderança, pensamento crítico, iniciativa, educação para o desenvolvimento sustentável entre outros. Em adição a isto, foram considerados os pontos levantados por Mills \& Treagust (2003), os quais afirmam que para se formar engenheiros para este novo tempo, é necessário superar as seguintes situações: a) os currículos de engenharia estão muito focados na ciência da engenharia e cursos técnicos, sem fornecer uma integração suficiente desses tópicos ou relacioná-los com a prática industrial. Os programas são orientados por conteúdo; b) os programas atuais não fornecem experiências de projeto suficientes para estudantes; c) os graduados ainda não possuem habilidades de comunicação e experiência de trabalho em equipe e os programas precisam incorporar mais oportunidades para que os alunos as desenvolvam; d) os programas precisam desenvolver mais consciência entre os estudantes sobre as questões sociais, ambientais, econômicas e legais que fazem parte da realidade prática de engenharia moderna; e) as faculdades existentes não possuem experiência prática, portanto não são capazes de, adequadamente reportar a teoria para praticar ou fornecer experiências de design; f) os programas estão desatualizados e precisam se tornar mais centrados no aluno.

O crescente aumento das exigências e responsabilidades dos profissionais da área tecnológica leva-nos a perceber que, para realizarem a sua prática com qualidade não basta apenas saber o conteúdo específico de sua matéria. O profissional necessita ter visão geral das potencialidades e problemas do país. Por essa razão, o profissional da área tecnológica não pode tratar tais situações como se fossem meros problemas instrumentais, susceptíveis de resolução através da aplicação de regras armazenadas no seu próprio conhecimento técnico científico, com apresentação do conceito de desenvolvimento desvinculados das condições históricas e sociais.

Educar ultrapassa a simples transmissão de conceitos, implica promover o desenvolvimento intelectual e social, de maneira a estimular a criatividade, a criticidade e a habilidade de aprender de forma constante e autônoma, acompanhando as mudanças da sociedade. O desenvolvimento científico-tecnológico induz a criação e o aperfeiçoamento de novos conhecimentos e técnicas, de suas aplicações e, consequentemente, exige amplos programas de formação de recursos humanos em seus vários níveis.

Desta forma, objetivando-se a promoção do ensino teórico de alta qualidade, alinhado à prática, indispensável aos estudantes, especialmente os de engenharia, e 
visando contribuir com a sociedade civil foi criado o Núcleo Integrado de Projetos de Engenharia (NIPE), o qual surgiu como alternativa para o atendimento às demandas referentes às engenharias (civil, elétrica, cartográfica, dentre outras), sendo estas comunitárias ou internas do campus. Este atendimento se dá, essencialmente, através da cooperação de alunos e professores das áreas técnicas (eletrotécnica, construção civil, agrimensura, saneamento) estando, portanto, alinhado com o proposto por Freire (1997), o qual afirma que para compreender a teoria é preciso experienciá-la. Essa afirmação é, empiricamente, verificada por um significativo percentual de estudantes que atribuem à experimentação um "caráter motivador, lúdico e essencialmente vinculado aos sentidos" (GIORDAN, 1999). Fato esse, ao menos no ensino de engenharia, que se torna vantajoso ao se considerar, por exemplo, os resultados de Felder e Spurlin (2005) mostrando, em dez diferentes instituições de ensino de engenharia, que os estudantes dessa área se apresentam mais sensoriais do que intuitivos.

\section{OBJETIVOS E ESPAÇO FÍSICO DO NIPE}

São objetivos do NIPE: Contribuir com o desenvolvimento da região a partir da geração de estágios que se transformem em projetos para atender demandas comunitárias e aquelas internas do campus, através do uso da carreta tecnológica como escritório modelo; Colaborar com a captação de recursos para execução dos projetos elaborados para o IFPA Campus Tucuruí ou para a comunidade externa de baixa renda; promover a capacitação dos estagiários e colaboradores, produção científica, maturação e experiência profissional aos alunos, integrando-os com docentes e comunidades diversas.

Ainda que o estágio seja elemento curricular obrigatório nos projetos pedagógicos de curso (PPCs), a quantidade de vagas de estágio disponíveis ainda é muito pequena na região de atuação do IFPA Tucuruí; somado a isto, a população de baixa renda presente na região não dispõe de recursos para custear despesas com elaboração de projetos técnicos de engenharia, e assim não consegue acessar recursos para o Terceiro Setor, a preocupação com a função social do conhecimento desenvolvida na academia é de suma importância, pois como afirma Foggiato (2007) Considerar a problemática do desenvolvimento científico e tecnológico, para que seja menos excludente é preciso que se levem em conta os reais problemas da população, os riscos técnico-produtivos e a mudança social. Por isso, faz-se necessário ter uma visão interativa e contextualizada das relações entre ciência, tecnologia, inovação e sociedade e, muito especialmente, de políticas públicas mais adequadas para gerenciar as oportunidades e perigos que envolvem uma mudança técnica.

Como é sabido, a crise econômica pela qual passa o país reduz as possibilidades do IFPA adquirir verbas diretas do governo para manter e melhorar as condições das atividades por ele desenvolvidas. Além do ensino oferecido, projetos de formação que buscam a excelência precisam capacitar seus profissionais e qualificá-los de acordo com suas afinidades individuais. O NIPE disponibiliza aos estagiários, cursos de capacitação e qualificação durante o período de extensão, que precede o período dos estágios.

O espaço físico do NIPE é constituído por uma carreta tecnológica adaptada e equipada com softwares e hardwares específicos para esse fim, que são de propriedade do IFPA Tucuruí. 
Figura 1- Espaço Interno da carreta tecnológica

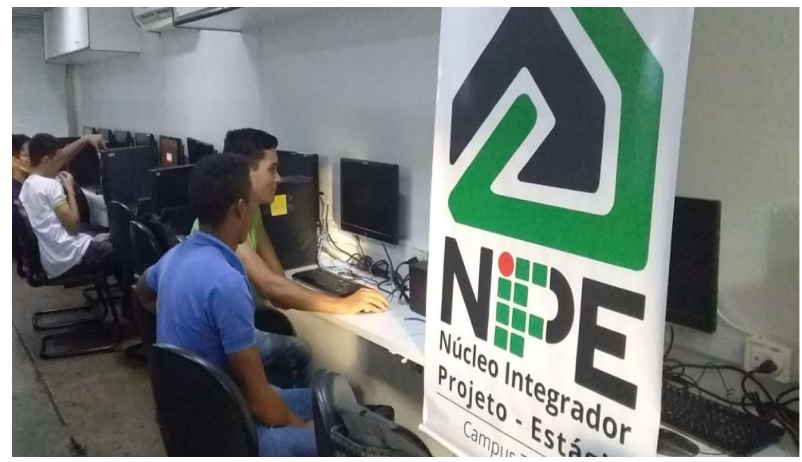

Fonte: Arquivo Autores

Figura 2- Espaço Interno da carreta tecnológica

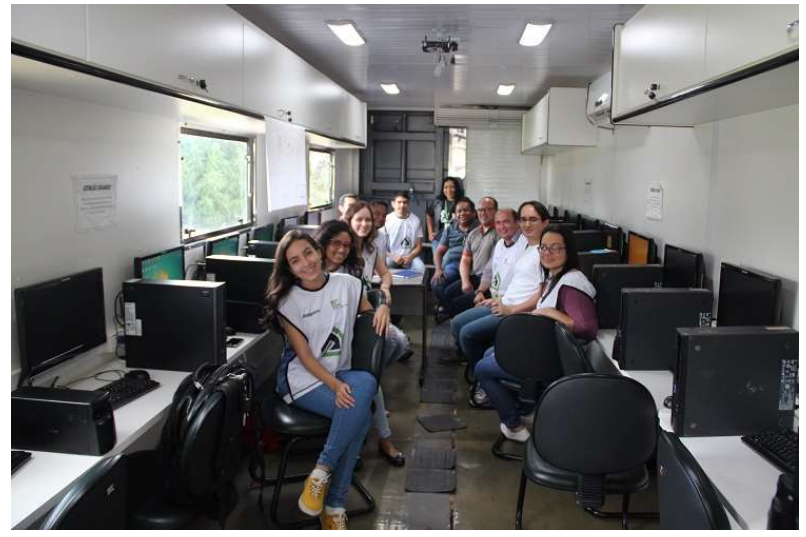

Fonte: Arquivo Autores

A troca de experiências e conhecimentos entre alunos, professores e comunidade, vem consolidando o NIPE como modelo inovador de escritório de projetos de engenharia que transforma aprendizado em experiências profissionais bem-sucedidas, visíveis nos resultados alcançados a partir de sua implantação em 2018. Portanto, o projeto se justifica não apenas agregar valores técnicos e sociais aos estagiários, futuros profissionais do mercado de trabalho, mas também por representar um avanço cultural significativo na formação humana, ao mesmo tempo em que busca por uma sociedade muito mais justa e igualitária. Para estimular o desempenho dos alunos nas disciplinas técnicas, o acesso aos estágios do NIPE é feito através de editais internos publicados no site do campus para os alunos do IFPA e através de processo de seleção para os alunos do IFPA e via entrevista direta para os alunos da UFPA, realizadas pelos professores envolvidos no projeto. Embora o mecanismo de acesso seja diferente para os alunos das duas instituições, a atuação dos discentes dentro do projeto é feita de forma conjunta, interagindo conhecimentos e partilhando cursos e uso de softwares e tecnologias disponíveis na carreta. Todas as ações realizadas no NIPE são supervisionadas pelo engenheiro do campus e pelos professores envolvidos no projeto específico. 


\section{PROJETOS DE ENGENHARIA DESENVOLVIDOS}

É importante destacar que todos os projetos aqui apresentados foram desenvolvidos integralmente dentro do NIPE, tendo efetiva e eficaz participação dos estagiários, obviamente sob a supervisão do corpo técnico e docente do Instituto, em todas as fases, a saber, projetos : arquitetônico, estrutural, de fundações, hidro sanitário, elétrico e lógico; tal metodologia ativa de aprendizagem é reconhecidamente efetiva na formação de estudantes : As metodologias ativas têm o potencial de despertar a curiosidade à medida que os alunos se inserem na teorização e trazem elementos novos, ainda não considerados nas aulas ou na própria perspectiva do professor (ANGELO;BERTONI, 2014; BERBEL, 2011).

Além da confecção de orçamento, cronograma físico financeiro e memorial descritivo, visto que, enquanto autarquia federal o IFPA está submetido à Lei de Licitações

\subsection{Projeto Ipirá}

Projeto de grandes proporções e média complexidade, foi proposto pelas Centrais Elétricas do Norte do Brasil (Eletronorte) em 2009 como compensação social às comunidades ribeirinhas devido aos impactos ambientais da construção da Usina Hidroelétrica de Tucuruí e Eclusas.

Constitui-se no maior projeto de piscicultura da região com ampla estrutura de engenharia. Convidado pelo Ministério Público Estadual, o IFPA solicitou ao NIPE o remodelamento de todos os projetos de engenharia, incluindo galpão depósito para ração, alojamento para os pescadores, rampa de acesso ao rio, sede administrativa e os projetos completos do Núcleo Didático de Aquicultura-NDA (Figura 3) o qual será contrapartida do Departamento Nacional de Trânsito (DNIT) para o IFPA. Todos os projetos, incluindo maquete eletrônica e orçamentos detalhados foram desenvolvidos pela equipe do NIPE.

Figura 3- Croqui de localização do projeto Ipirá

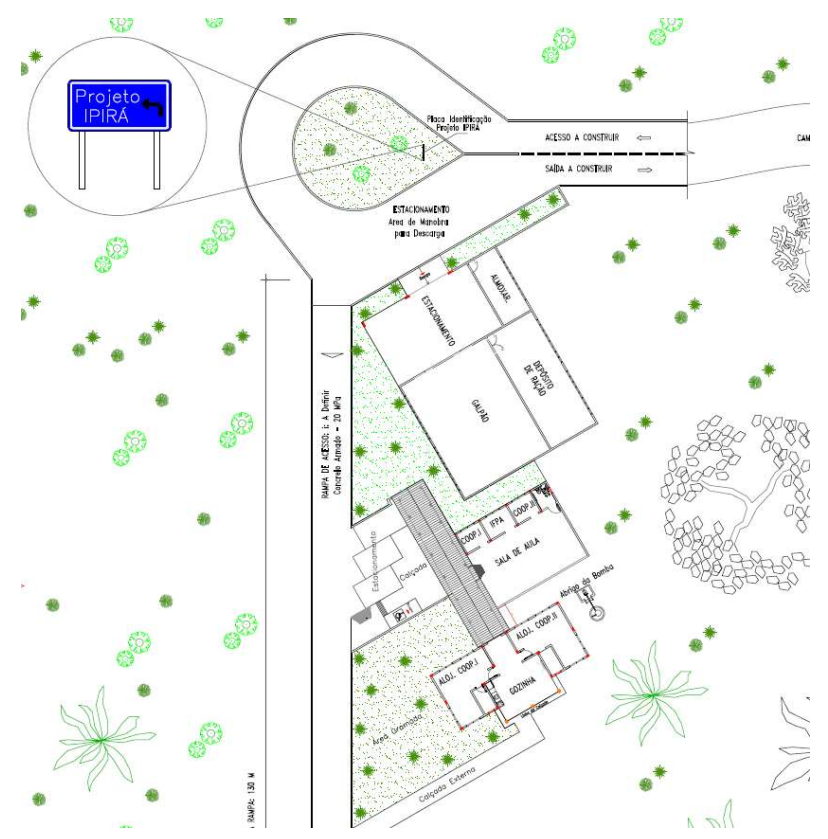

Fonte: Arquivo Autores 
Figura 4- Detalhe arquitetônico do depósito de ração

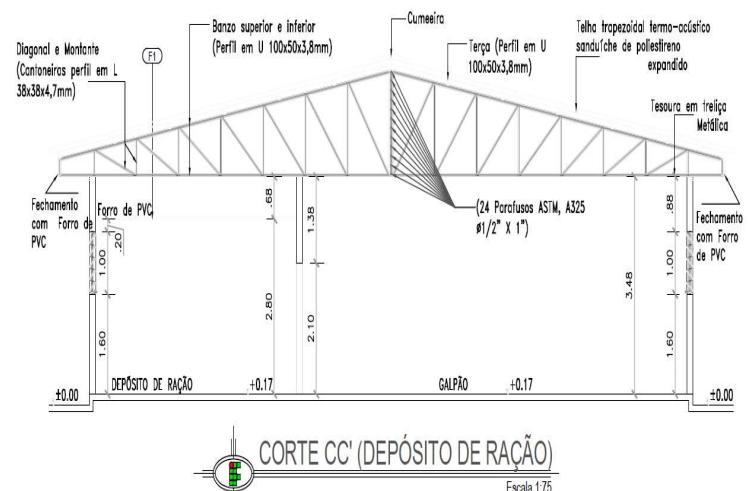

Fonte: Arquivo Autores

\subsection{Projeto do restaurante universitário}

Projeto fundamental para a melhoria da reestruturação do novo Campus do IFPA, o qual localiza-se na Avenida Brasília, e melhor conforto para toda a comunidade. Totalmente desenvolvido no NIPE, o projeto completo incluiu também Maquete eletrônica em 3D, Orçamentos detalhados, Memoriais Descritivos e Especificações Técnicas dos materiais; além do atendimento às especificidades de natureza sanitária, inerentes à cozinha industrial, cujo projeto foi desenvolvido de modo concomitante ao do restaurante universitário, com participação direta dos técnicos administrativos do setor de nutrição. Estes projetos serão encaminhados para fontes de recursos na busca por recursos para a sua execução.

Figura 5- Detalhe arquitetônico do depósito de ração

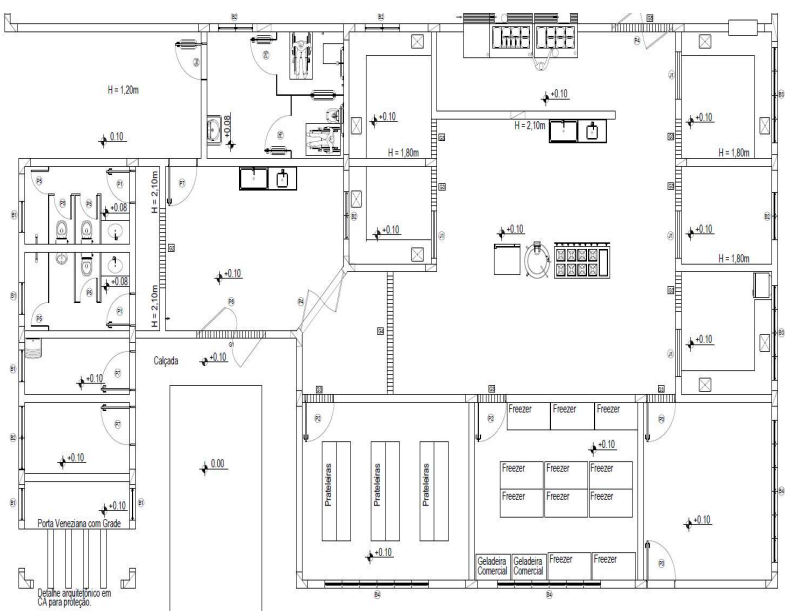

Fonte: Arquivo Autores

Figura 6- Maquete 3D do Restaurante 


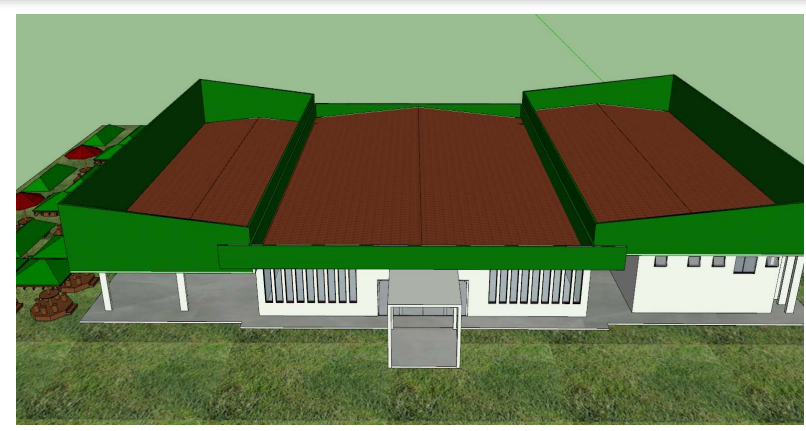

Fonte: Arquivo Autores

\section{Elaboração de Artigos Científicos}

A partir dos trabalhos e projetos realizados durantes os estágios no NIPE, somados aos projetos integradores, ao quais constituem-se em componentes curriculares obrigatórios na formação técnica, a equipe formada por professores e alunos foi constituída, com o intuito de elaborar artigos acadêmicos que possam ser publicados apresentando a comunidade científica os resultados desses trabalhos, corroborando com os ideais de divulgação e popularização da ciência, mais especificamente no que concerne ao conhecimento produzido e desenvolvido no ambiente de ensino das engenharias e cursos técnicos correlatos.

\subsection{Painel do NIPE}

Estando alinhados coma tríade: ensino, pesquisa e extensão; e fundamentado no princípio de popularização do conhecimento, e como forma de apresentar à comunidade acadêmica local e a sociedade em geral, aqueles trabalhos de maior relevância desenvolvidos, um evento denominado Painel do NIPE é realizado a cada final de semestre, oportunidade em que são apresentados Projetos de Engenharia, Projetos Integradores e a entrega dos certificados dos estagiários, cursos e extensionistas concluintes.

Figura 7- Certificação de conclusão de estágio e cursos no segundo painel do NIPE

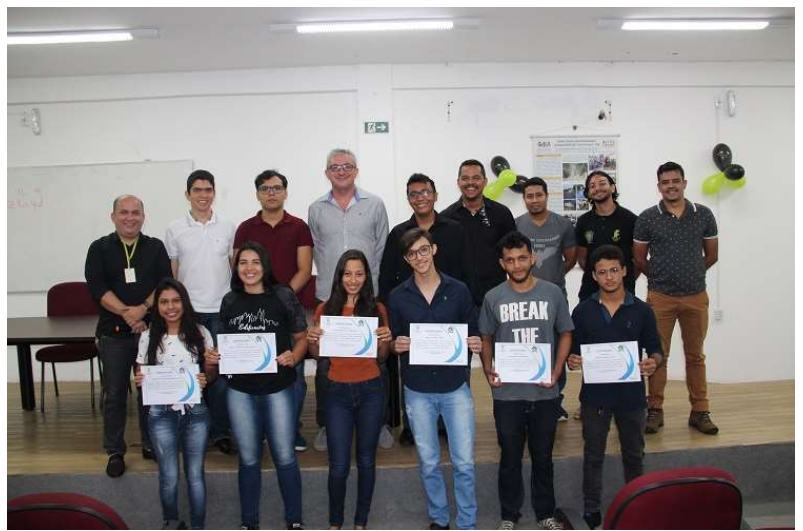

Fonte: Arquivo Autores 
Figura 8- Certificação de conclusão de estágio e cursos no primeiro painel do NIPE

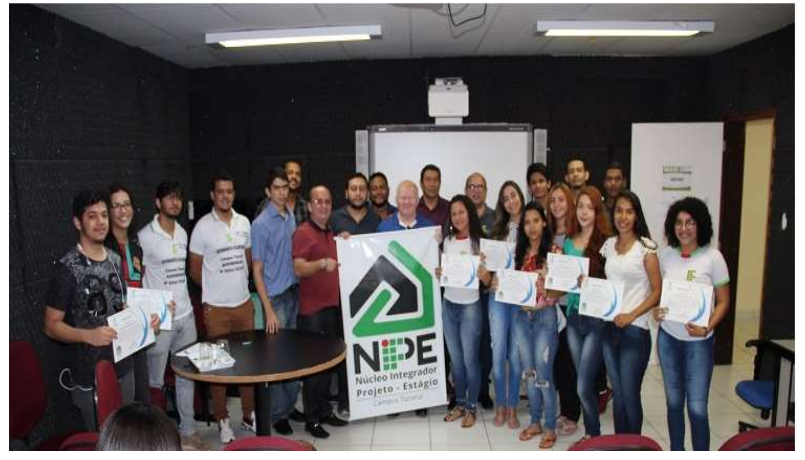

Fonte: Arquivo Autores

Durante a realização do segundo painel do NIPE, estando cientes das especificidades e importância decorrentes da divulgação do Decreto Federal 9.377/2018, o qual traz a obrigatoriedade para que todos os projetos de engenharia do Brasil passem a acontecer a partir de 2021, dentro da Plataforma BIM (Building Information Modeling), como parte da estratégia nacional de incentivo ao uso dessa tecnologia. Foi informado aos presentes que, no NIPE já está em curso, através da disponibilização do software e cursos específicos neste ambiente, a busca por excelência para a equipe de alunos, professores e para profissionais de engenharia de modo geral que atua diariamente nas ações desenvolvidas na carreta.

\section{$5 \quad$ Considerações FINAIS}

Esta metodologia envolve estudantes trabalhando em equipes, visando elaborar projetos e resolver problemas concretos e reais de engenharia, utilizando a teoria para aplicação em ações práticas. Esse é um dos aspectos positivos da aplicação de tal metodologia, uma vez que na atuação de sua futura profissão, provavelmente, engenheiros e técnicos irão trabalhar em equipes, sendo, portanto, importante que aprendam como se relacionar e entendam as dificuldades de se trabalhar em grupos.

O uso de metodologias de ensino ativa também levou a resultados positivos dentro de sala de aula, pois notou-se que os conhecimentos produzidos em sala fossem muito bem aproveitados e gerassem um resultado final melhor do que o esperado. Percebeu-se que os estudantes estavam mais motivados e participativos quando comparados com as aulas regulares expositivas ministradas anteriormente.

Somado a estes fatos, a efetiva conclusão dos projetos e obras mencionados no artigo, estando estes de acordo com as normas técnicas vigentes, nos possibilita concluir que o NIPE vem alcançando sucesso em seus objetivos de promover a integração teórica e prática do ensino, além de servir à comunidade da região de Tucuruí.

\section{REFERÊNCIAS}

\section{Livros}

BERBEL, N. A. N. As metodologias ativas e a promoção da autonomia de estudantes. Ciências Sociais e Humanas, Londrina, v. 32, n. 1, p. 25-40,jan./jun. 2011. CUNHA, M. L. R. et al. Evaluation of student perceptionof the Team-based Learning method (APATBL):Instrument construction and validation.

FOGGIATTO,R. Educação tecnológica: qual o seu papel. Anais...XXXV Congresso Brasileiro de Educação em Engenharia, Curitiba, 2007. 
FREIRE, P. Pedagogia da autonomia. Rio de Janeiro:Paz e Terra, 1997.

\title{
Capítulos de Livros:
}

FELDER, R. M.; SPURLIN, J. Reliability and validity of the Index of Learning Styles: a meta-analysis International Journal of Engineering Education.v. 21, n. 1, p. 103-112, 2005.

\section{Monografias, dissertações e teses:}

GIORDAN, M. O papel da experimentação no ensinode ciências. Química nova na escola, online, n.10, 1999. Disponível em: <http://qnesc.sbq.org.br/online/qnesc10/pesquisa.pdf>. Acesso em: 18 jan.2017.

Learning Theanswer? The Australasian Associationfor Engineering Education Inc (2003).Disponível m: http://www.aaee.com.au/journal/2003/mills_treagust03.pdf $>$. Acesso em: 22 abr.2019.

MILLS, J. E.; TREAGUST D. F. Engineering Education- Is Problem-Based or Project-Based Learning Theanswer? The Australasian Associationfor Engineering Education Inc (2003).Disponível m: http://www.aaee.com.au/journal/2003/mills treagust03.pdf $>$. Acesso em: 22 abr.2019.

\begin{abstract}
The Integrated Nucleus of Projects and Engineering (NIPE) was conceived as a project office, seeking to unify teaching, research and extension, whose operation occurs in an accessible cart, equipped with technology and software for wide commercial use for the production of engineering projects. Extension project proposed by some professors at the Federal Institute of Pará, Campus Tucuruí, provides internships for students of technical and higher courses at the institution itself, and engineering students at the Federal University of Pará who. Based on these technologies, they elaborate projects oriented and coordinated by professors-engineers and by the effective engineer of the Campus. This article aims to report on the objectives and projects developed at NIPE, as well as to demonstrate the importance and effectiveness of using this active methodology in students' learning about engineering and technological education, in addition to aggregating and generating knowledge and benefits for the academic community and community generally. The application of this active methodology supervised by the teaching and technical staff, observed a visible increase in student engagement and provided a huge improvement in practical learning results and transversal skills, such as time management, interpersonal communication and autonomy in decisions. One of the main positive points observed was the students' motivation, when they were able to associate the projects developed in the office with professional situations, managing to relate theory \& practice to the real context. The team interaction made it possible to conclude interesting engineering projects that will be effectively built on the campus and in the city of Tucuruí itself, and it can be said that NIPE achieved its objectives as a means of promoting teaching, research and extension and generating internships and collective benefits.
\end{abstract}

Keywords: Active Methodology. Teaching by experimentation. Technology. Office. Engineering Projects. 